\title{
Foreword to the special issue on recent advances on pattern recognition and artificial intelligence
}

\author{
Huawen Liu ${ }^{1}$ Jianping $\mathrm{Yin}^{2} \cdot$ Xudong Luo ${ }^{3}$ Shichao Zhang ${ }^{4}$
}

(C) The Natural Computing Applications Forum 2017

The recent years have witnessed the great progresses of artificial intelligence and its applications in a wide range of fields. Particularly, there has been an increasing interest in artificial intelligence combined with pattern recognition techniques, to build learning models that solve complicated problems in different fields. As we know, pattern recognition has its roots in artificial intelligence, engineering and statistics. It concerns with the problems of identification and recognition of patterns and regularities in data. Pattern recognition is often considered as a branch of machine learning, and in some cases they are mistaken to each other. It is interdisciplinary and extensively studied in many fields, including computer science, statistics, psychology, linguistics, philosophy, cognitive science, as well as other

Huawen Liu

hwliu@zjnu.edu.cn

Jianping Yin

jpyin@nudt.edu.cn

Xudong Luo

luoxd3@mail.sysu.edu.cn

Shichao Zhang

zhangsc@gxnu.edu.cn

1 Department of Computer Science, Zhejiang Normal University, 688 Yinbing Rd, Jinhua 321004, People's Republic of China

2 School of Computer Science, National University of Defense Technology, 109 Deya Rd, Changsa 410073, People's Republic of China

3 Department of Philosophy, Sun Yat-sen University, 135 Xingang Xi Rd, Guangzhou 510275, People's Republic of China

4 School of Computer Science and Information Engineering, Guangxi Normal University, 15 Yucai Rd, Guilin 541004, People's Republic of China specialized fields such as ethology. Pattern recognition is a rich family of learning methods, including classification, clustering, discriminative analysis, regression, dimension reduction, and has now been widely applied in a great number of domains, including image processing, text categories, computer vision, robotics and speech recognition.

During past years, many scientists have endeavored to pattern recognition and artificial intelligence. In particular, recent emerging techniques, including deep learning, big data and social computing, greatly advance progresses in pattern recognition and artificial intelligence. These methods are the subject of a recent surge of interest of pattern recognition for two main reasons: new technologies and new applications. By now, a great number of conferences and workshops on pattern recognition and related fields were organized by mathematical and computer scientists, showing the continued flourishing and fruitful research of pattern recognition and artificial intelligence, and their applications. However, there are also some debates as to the reasons for the success of these methods, and about their limitations. In fact, there are no widely acceptable answers for open questions, such that how they can be tailored to certain practicable applications and how they can take advantage of massively computing devices.

This special issue is dedicated to bring together research work in the areas of pattern recognition and artificial intelligence, thereby providing a bird's eye view of the developing landscape of the aforesaid fields and their related applications today, and a consolidated foundation to the interested researchers for further research in theoretical and practical aspects. Each paper was reviewed by at least three reviewers from the communities of pattern recognition and artificial intelligence around the world, with at least two rounds before acceptance. 
We hope that the readers will find the papers within this special issue, which covers multidisciplinary domains, interesting and that further pursuits will be engaged in pattern recognition and artificial intelligence, in theoretical and engineering manner.

Finally, the guest editors wish to give our deep gratitude to all the authors that contributed to this special issue. We would like to the reviewers for their insightful and valuable reviews of manuscripts, providing timely review reports to improve the works. We are also grateful to thank the Editor-in-Chief, Professor John MacIntyre, and editorial office of Neural Computing and Applications for their support and giving us the opportunity to publish this special issue. 